\title{
Adultos livres de cárie: estudo de casos e controles sobre conhecimentos, atitudes e práticas preventivas
}

\author{
Adults free of caries: a case-control study about: \\ awareness/consciousness, attitudes \\ and preventive practices
}

Paulo C. Petry 1

César G. Victora 2

Iná S. Santos 2

\footnotetext{
1 Faculdade de Odontologia, Universidade Federal do Rio Grande do Sul. Rua Ramiro Barcel os 2492, Porto Al egre, RS 90035-003, Brasil. 2 Centro de Pesquisas em Epidemiologia, Universidade Federal de Pelotas. Rua Duque de Caxias 250, Pelotas, RS 96030-002, Brasil
}

Abstract The purpose of this article was to investigate the effect of personal awareness and attitudes, the dentist's role, oral hygiene habits, and fluoride use on total absence of caries in adults, using a case-control study matched for sex and age with undergraduate students from two private Brazilian universities. Crude analysis using conditional logistic regression failed to show any effect for the related variables regarding fluoride use and oral hygiene habits $(p>0.20)$. Use of dental floss was the only exception in this set. It appeared as a risk factor, since individuals who stated not flossing presented a crude odds ratio (OR) of 0.70 , or 0.73 when adjusted for regular visits to the dentist. The effect for regular visits to the dentist was that of a risk. Those with a history of caries presented a crude odds ratio of 1.55; after adjusting for dental floss use, the risk was slightly higher (OR =1.67). Oral hygiene habits, contrary to the formulated hypothesis, did not display the expected protective effect. Likewise, fluoride use was not significantly associated with absence of caries. Individuals with a history of caries were used to visiting more professionals, confirming the formulated hypothesis for this variable. Nevertheless, it is still intriguing that some people fail to devel op caries, even when they report not practicing measures considered ideal for oral health.

Key words Dental Caries; Preventive Dentistry; Oral Heal th; Dentistry

Resumo Com o objetivo de investigar o efei to do conhecimento e atitudes pessoais, hábi tos de higi ene oral e uso de fluoretos sobre a total ausência de cáries, realizou-se um estudo do ti po caso-controle entre estudantes uni versitários da regi ão sul do Brasil. A análise bruta por regressão logística condicional não demonstrou efei to para as variáveis relacionadas ao uso defluoretos e hábitos de hi giene oral $(p>0,20)$. Já o uso de fio dental apareceu como um fator de risco: os que afirmaram não usá-lo apresentaram “odds rati o" (OR) bruto de 0,70, e de 0,73 quando ajustado para visitas regulares ao dentista. O efeito para as visitas regulares ao dentista foi de risco. Pessoas com história de cárie visi tavam mais os profissi onais. Aqueles com história de cárie apresentaram um "odds ratio" bruto de 1,55; após o ajuste para uso de fio dental o risco foi levemente superior ( $O R=1,67$ ). Hábitos de higi ene oral não demonstraram efeito protetor esperado. $O$ uso de fluoretos não esteve significati vamente associ ado à ausênci a de cáries. Permanece intrigando o fato de que al gumas pessoas não desenvol vam cáries, mesmo não relatando cuidados consi derados ideais à saúde bucal.

Palavras-chave Cárie Dentária; Odontologia Preventiva; Saúde Bucal; Odontologia 
Introdução

Não há dúvidas quanto à importância de uma prática odontológica que contemple a saúde bucal coletiva, universalizando o acesso e garantindo a eqüidade no atendimento das necessidades da população. É marcante o declínio recente na experiência da doença cárie, em especial, na população jovem de países industrializados (Stamm, 1991; Thylstrup \& Bruun, 1992). Na Inglaterra e no País de Gales, de 1968 a 1988, o número de adultos com dentes aumentou em $41 \%$ e o número de dentes sadios, em 65\% (Downer, 1991). Na Dinamarca, desde 1978, houve redução média de $69 \%$ de dentes cariados em crianças de 5 anos de idade e de aproximadamente $75 \%$, entre crianças de 12 anos. Em quatro países nórdicos (Dinamarca, Finlândia, Noruega e Suécia), a proporção de crianças livres de cárie, em 1988, era de cerca de $50 \%$ aos 5 anos e de $25 \%$ aos 12 anos, evidenciando nítido declínio nos níveis de doença (Fehr, 1990). As maiores reduções ocorreram onde foram oferecidos programas coletivos amplos de atenção às crianças (Fejerskov et al., 1982).

Semelhantes programas preventivos são pouco comuns nos países em desenvolvimento, justamente onde são mai ores os problemas de saúde bucal e menores as ofertas de serviços curativos. No Brasil, sabe-se que a odontologia curativa - por seu caráter privado, individualista e de altos custos - tornou-se inacessível a cerca de $90 \%$ da população brasileira. (Cordón \& Garrafa, 1991).

Por sua vez, a mercantilização da prevenção também torna a assistência preventiva privilégio para poucos. Neste país estima-se que sejam produzidas anualmente cerca de 52 toneladas de dentifrícios, vendidas por volta de 75 milhões de escovas dentais e consumidos ao redor de 1 bilhão de metros de fio dental (Istoé/Senhor, 1988; Odontonotícias, 1988, 1992), dados que revelam a flagrante inadequação do consumo para uma população de 160 milhões de habitantes. Também é grave o fato de que, em função das desigualdades sociais e da má distribuição de renda, a mesma camada da sociedade a beneficiar-se dos serviços odontológicos seja a que consome estes produtos de higiene bucal.

No Brasil, não existem estudos a respeito de pessoas que atingem a idade adulta sem desenvolver cáries. Em outros países, avalia-se que seja de $1 \%$ a $2 \%$, no máximo, a sua prevalência (Nikiforuk, 1985). Por intermédio do presente estudo, pretende-se investigar se os aspectos ligados à prática odontológica no país estão relacionados à ausência de história de cáries em adultos. Por exemplo, será a total ausência de cáries decorrente da prevenção? Por que certas pessoas não desenvolvem cáries? Possíveis respostas a essas indagações poderão ampliar o nível de conhecimento a respeito do processo de determinação da doença, bem como contribuir para a implementação de políticas de saúde bucal coletivas capazes de produzir efeitos em todas as camadas sociais.

O presente estudo de casos e controles (Schlesselman, 1982) visa analisar a importância de fatores específicos - higiene oral e uso de fluoretos, conhecimento e atitudes pessoais, e o papel do dentista - para que se chegue à idade adulta livre de cáries.

\section{Metodologia}

Para o entendimento do processo saúde-doença cárie sob a óptica da multicausalidade (Victora et al., 1994) é imprescindível construir-se modelo teórico ou conceitual (Facchini, 1995). A construção de tal modelo para o estudo de pessoas livres de cárie foi descrita anteriormente (Petry et al., 1996) e está representada na Figura 1. Fatores sócio-econômicos, genéticos e familiares encontram-se em primeiro nível hierárquico de causalidade e podem influenciar os fatores localizados em níveis inferiores. No segundo nível, encontram-se fatores comportamentais (como o tabagismo e o uso de álcool), o papel do dentista, além de conhecimentos e atitudes relacionados à saúde bucal. A seguir, em terceiro nível e influenciados pelas variáveis acima, vêm os hábitos dietéticos, de higiene oral e o uso de fluoretos, os quais constituem os determinantes proximais (intrabucais) da ocorrência de cáries. Estas variáveis exercem efeito direto na presença ou ausência de cáries, ao mesmo tempo em que intermedeiam os efeitos dos fatores anteriores.

\section{Seleção de casos e controles}

Nos delineamentos clássi cos de casos e controles, os casos são os portadores da doença em estudo (Schlesselman, 1982). Em geral, as doenças enfocadas são raras e, desta forma, há excesso de controles em relação aos casos. Desse modo, para cada caso, seleciona-se um ou mais controles entre as pessoas livres da doença. Contudo, no atual estudo procede-se ao contrário, pois as pessoas livres da doença cárie eram poucas e constituíram o grupo dos casos, ao passo que seus controles deveriam apresentar atividade presente e/ ou passada de cáries. 
Para inclusão no estudo foram avaliados alunos matriculados nos diferentes cursos de graduação da Universidade Luterana do Brasil (ULBRA), Canoas, Rio Grande do Sul e Universidade do Vale do Itajaí (UNIVALI), Itajaí, Santa Catarina. Ambas são universidades privadas e localizam-se em regiões desenvolvidas do Brasil. O trabal ho de coleta dos dados foi executado por seis alunos dos Cursos de Odontologia previamente treinados para a realização dos exames clínicos.

Com o objetivo de identificar as pessoas livres de cárie (potenciais casos), perguntou-se, nas salas de aula, pela existência de alunos que nunca haviam restaurado seus dentes. A confirmação da condição de caso foi feita por meio de exame clínico bucal recomendado pela Organização Mundial da Saúde (WHO, 1987), selecionando-se apenas indivíduos sem qualquer tipo de restauração ou sinais de atividade de cárie, presente ou passada, como manchas brancas ativas ou inativas e cavidades cariosas.

Na mesma sala de aula, foram escolhidos, para cada caso, dois controles que pertencessem ao mesmo sexo e tivessem a data de aniversário mais próxima à do caso (emparelhamento para sexo e idade). De modo a integrar o grupo controle, os alunos foram submetidos a idêntico exame bucal, devendo apresentar restaurações e/ ou sinais clínicos de atividade cariosa.

\section{Variáveis estudadas}

Imediatamente após a realização dos exames bucais foram entregues, a casos e controles, questionários auto-aplicáveis, padronizados e pré-codificados, os quais continham questões relativas às seguintes variáveis:

- Biológicas: sexo e idade;

- Papel do Cirurgião dentista: regularidade das consultas: costume de consultar regularmente o dentista; freqüência das consultas: entre os que consultam regularmente, qual o intervalo de tempo em que o fazem; recomendação da pasta dental: se o dentista orientou quanto à escolha da pasta dental; orientação sobre higiene oral: se o dentista orientou a respeito da maneira de higienizar a boca;

- Conhecimento e atitudes pessoais: compra da escova dental: se feita pelo próprio usuário ou por outra pessoa; nome da pasta: se o respondente sabe o nome da pasta que está usando; pasta fluoretada: se o entrevistado sabe se a pasta contém flúor;

- Hábitos de higiene oral e uso de fluoretos: escovação dental: qual o número de escovações diárias e se são feitas escovações fora do am-
Figura 1

Modelo teórico conceitual hierarquizado

entre os fatores em estudo.

Nível distal de determinação

Variáveis sociais, genéticas e familiares

Nível socioeconômico

Escolaridade dos pais

Fonte de água na infância

Existência de familiar livre de cáries

Presença de dentista na família

Nível intermediário de determinação

Variáveis comportamentais

Hábito de fumar

Consumo de bebidas alcoólicas

Papel do Dentista

Conhecimentos e atitudes pessoais

Nível proximal de determinação

Variáveis comportamentais

Hábitos dietéticos

Uso de fluoretos

Higiene oral

Desfecho

Ausência de cáries

biente doméstico; uso de fio dental: se há uso rotineiro e qual a freqüência de uso; uso de flúor: se há uso rotineiro e qual o veículo de aplicação.

Além das variáveis acima, duas outras foram incluídas na análise - por terem sido identificadas em estudo anterior como associadas com estar livre de cáries - como possíveis fatores de confusão: presença de al gum familiar livre de cáries e freqüência de consumo de açúcares (Petry et al., 1996).

- Fatores sócio-econômicos, familiares e dietéticos: fonte de abastecimento de água na infância: encanada ou outras; escolaridade dos pais: ambos com 1o grau completo, ambos com 3o grau completo ou outras combinações; escore de bens sofisticados (forno de microondas, telefone ecomputador): posse de três, dois, um ou nenhum dos bens; presença de dentista na família: em qualquer grau de parentesco; existência de familiar livre de cáries: em qualquer grau de parentesco; número de refeições diárias: hábito de lanchar entre as refeições; tipo e freqüência de lanches; consumo habitual de 
balas, chicletes e refrigerantes; tipo de adoçante preferido e escore de consumo freqüente de açúcares.

\section{Tamanho da amostra}

Para o cálculo do tamanho da amostra foram adotados os seguintes parâmetros:

- Nível de significância estatística de 95\% (erro alfa de $5 \%$ );

- Poder do estudo de 80\%;

- Dois controles para cada caso;

- Freqüência esperada de exposição entre os controles de $35 \%$;

- Odds ratio de 2,00.

Ao resultado obtido (327 pessoas), acrescentaram-se $15 \%$ para o controle de possíveis fatores de confusão. A amostra final do estudo foi constituída por 378 pessoas, dentre as quais havia 126 casos e 252 controles.

A nálise

Para a entrada de dados utilizou-se o programa Epi Info, versão 6.02 (Epi Info, 1994). As tabulações univariadas e a análise multivariada - conforme modelo hierarquizado (Figura 1) - por regressão logística condicional (Breslow \& Day, 1980) foram realizadas com o programa SPSS for Windows (SPSS for Windows, 1994).

No processo de análise multivariada, permaneceram no modelo apenas as variáveis que apresentavam significância estatística para determinado nível. Adotou-se, para este procedimento, um valor de p menor ou igual a 0,20, de modo a evitar a exclusão de variáveis potencialmente importantes (Maldonado \& Greenland, 1993). Isto implica que al guns dos intervalos de confiança (IC 95\%) incluíram a unidade.

\section{Resultados}

\section{A nálise univariada}

Um total de 126 casos e 252 controles foi incluído no estudo, do qual 56,3\% pertenciam à UNIVALI, Santa Catarina. As médias de idade foram similares entre casos ( 20,5 anos) e controles (20,6 anos), ressaltando-se que, em cada grupo, 57,1\% eram mulheres.

A distribuição das principais variáveis entre pessoas livres de cárie (casos) e com história de cárie (controles) é apresentada na Tabela 1.

Embora a maioria dos casos visite regularmente o dentista, por volta de 35\% não o fazem. Entre os controles, apenas cerca de $20 \%$ não são freqüentadores regulares de consultó- rios odontológicos. Entre os que consultam regularmente, $33 \%$ dos casos e $25 \%$ dos controles somente o fazem uma vez ao ano. Afirmaram fazer três visitas anuais, $20 \%$ dos casos e $28 \%$ dos controles.

A participação do dentista na escolha da pasta dental é baixa. Em ambos os grupos, cerca de $80 \%$ das pessoas informaram não ter recebido qualquer recomendação a esse respeito. Quanto à compra da própria escova dental, $18 \%$ dos casos asseveraram que não o fazem, contra $7 \%$ dos controles. No que concerne ao conhecimento a respeito da pasta dental, $11 \%$ dos casos e $10 \%$ dos controles disseram não saber a marca comercial que usam. Além disso, 9,5\% dos casos e $13,1 \%$ dos controles declararam não saber se a mesma contém flúor.

Cerca de metade das pessoas nos dois grupos escova os dentes três vezes ao dia. Entre os casos, $18,5 \%$ fazem apenas uma ou duas escovações diárias e $28 \%$ escovam quatro vezes ou mais por dia. Entre os controles, essas proporções foram de $13 \%$ e $35 \%$, respectivamente. Quando passam o dia na Faculdade, 43\% dos casos e $35 \%$ dos controles não escovam os dentes após a refeição.

Apenas $44 \%$ dos casos sustentaram usar fio dental; destes, 81\% já tinham recebido orientação quanto a seu uso correto. Entre os controles, $62 \%$ usam o fio e $84 \%$ tinham recebido orientação para este fim. Tal orientação foi dada pelo cirurgião dentista para cerca de $70 \%$ tanto dos casos quanto dos controles.

A utilização de flúor é feita somente via pasta dental entre aproximadamente $70 \%$ das pessoas de ambos os grupos. Apenas $26 \%$ dos livres de cárie e $30 \%$ dos indivíduos com história de cárie usam outros métodos de aplicação de fluoretos.

A Tabela 2 mostra os resultados da análise univariada por regressão logística condicional. Dos fatores relativos ao papel do dentista, observou-se que o efeito de indicar a pasta dental ( $p=0,76)$ e a freqüência de consultas ( $p=0,51)$ não estiveram significativamente associados à ausência de cáries. Tampouco foram significativas as variáveis relacionadas ao conhecimento e às atitudes pessoais em saúde bucal, todas com valor de $p$ superior a 0,21. As variáveis relativas aos cuidados de higiene oral - escovação dental diária $(p=0,42)$, escovação dental na Faculdade ou trabalho ( $p=0,50)$, uso do fio dental $(p=0,21)$ e uso de fluoretos $(p=0,54)$ também não estiveram associadas ao desfecho.

Com relação às consultas regulares, registrou-se associação estatisticamente significativa $(p=0,02)$. Paradoxal mente, indivíduos com história de cáries afirmaram visitar com maior 


\section{Tabela 1}

Distribuição das variáveis em estudo entre pessoas livres de cárie (casos) e com história de cáries (controles).

\begin{tabular}{|c|c|c|}
\hline Variável & $\begin{array}{c}\text { Casos }(\%) \\
n=126\end{array}$ & $\begin{array}{c}\text { Controles (\%) } \\
n=252\end{array}$ \\
\hline \multicolumn{3}{|c|}{ Freqüência de visitas ao dentista } \\
\hline $1 \mathrm{vez} / \mathrm{ano}$ & $24(32,9)$ & $45(25,0)$ \\
\hline 2 vezes/ano & $34(46,6)$ & $85(47,2)$ \\
\hline 3 vezes/ano & $15(20,5)$ & $50(27,8)$ \\
\hline \multicolumn{3}{|c|}{ Visitas regulares ao dentista } \\
\hline Sim & $82(65,1)$ & $203(80,6)$ \\
\hline Não & $44(34,9)$ & $49(19,4)$ \\
\hline \multicolumn{3}{|c|}{ Indicação de pasta pelo dentista } \\
\hline Sim & $25(21,9)$ & $41(19,0)$ \\
\hline Não & $89(78,1)$ & $175(81,0)$ \\
\hline \multicolumn{3}{|c|}{ Compra da escova dental } \\
\hline Sim & $103(81,7)$ & $234(92,9)$ \\
\hline Não & $23(18,3)$ & $18(7,1)$ \\
\hline \multicolumn{3}{|c|}{ Sabe o nome da pasta dental } \\
\hline Sim & $112(88,9)$ & $223(89,6)$ \\
\hline Não & $14(11,1)$ & $26(10,4)$ \\
\hline \multicolumn{3}{|c|}{ Sabe se a pasta tem flúor } \\
\hline Sim & $114(90,5)$ & $219(86,9)$ \\
\hline Não & $12(9,5)$ & $33(13,1)$ \\
\hline \multicolumn{3}{|c|}{ O rientação uso fio dental } \\
\hline Sim & $101(80,8)$ & $212(84,1)$ \\
\hline Não & $24(19,2)$ & $40(15,9)$ \\
\hline \multicolumn{3}{|c|}{ Quem ensinou uso fio dental } \\
\hline Dentista & $67(67,0)$ & $158(74,9)$ \\
\hline Familiares & $33(33,0)$ & $53(25,1)$ \\
\hline \multicolumn{3}{|l|}{ Escovações diárias } \\
\hline 1 ou 2 & $33(18,5)$ & $32(12,9)$ \\
\hline 3 & $66(53,2)$ & $130(52,2)$ \\
\hline 4 ou mais & $35(28,2)$ & $87(34,9)$ \\
\hline \multicolumn{3}{|c|}{$\begin{array}{l}\text { Escovação na faculdade } \\
\text { ou trabalho }\end{array}$} \\
\hline Sim & $46(36,5)$ & $103(40,9)$ \\
\hline Às vezes & $26(20,6)$ & $62(24,6)$ \\
\hline Não & $54(42,9)$ & $87(34,5)$ \\
\hline \multicolumn{3}{|l|}{ Uso do fio dental } \\
\hline Sim & $55(43,7)$ & $156(61,9)$ \\
\hline Às vezes e/ou não & $71(56,3)$ & $96(38,1)$ \\
\hline \multicolumn{3}{|c|}{ Uso do flúor além da pasta dental } \\
\hline Sim & $33(26,2)$ & $76(30,3)$ \\
\hline Não & $93(73,8)$ & $175(69,7)$ \\
\hline
\end{tabular}


Efeitos brutos das variáveis relacionadas ao papel do dentista, conhecimento, atitudes pessoais, hábitos de higiene oral e uso de fluoretos. Análise por regressão logística condicional.

\begin{tabular}{|c|c|c|}
\hline Variável & $\begin{array}{l}\text { OR brutoA } \\
\text { (IC 95\%)B }\end{array}$ & $p$ valor \\
\hline \multicolumn{3}{|c|}{ Freqüência de visitas ao dentista } \\
\hline 1 vez/ano & 1,00 & 0,51 \\
\hline 2 vezes/ano & $0,88(0,54-1,44)$ & \\
\hline 3 vezes/ano & $0,72(0,42-1,25)$ & \\
\hline \multicolumn{3}{|c|}{ Visitas regulares ao dentista } \\
\hline Não & 1,00 & $0,02 *$ \\
\hline Sim & $0,64(0,44-0,93)$ & \\
\hline \multicolumn{3}{|c|}{ Indicação de pasta pelo dentista } \\
\hline Sim & 1,00 & 0,76 \\
\hline Não & $0,93(0,60-1,42)$ & \\
\hline \multicolumn{3}{|c|}{ Compra da escova dental } \\
\hline Sim & 1,00 & $0,02 *$ \\
\hline Não & $1,92(1,11-3,33)$ & \\
\hline \multicolumn{3}{|c|}{ Sabe o nome da pasta dental } \\
\hline Sim & 1,00 & 0,46 \\
\hline Não & $1,03(0,62-1,72)$ & \\
\hline \multicolumn{3}{|c|}{ Sabe se a pasta tem flúor } \\
\hline Sim & 1,00 & 0,46 \\
\hline Não & $0,83(0,51-1,35)$ & \\
\hline \multicolumn{3}{|c|}{ O rientação sobre uso do fio dental } \\
\hline Sim & 1,00 & 0,21 \\
\hline Não & $1,14(0,75-1,75)$ & \\
\hline \multicolumn{3}{|c|}{ Quem ensinou o uso do fio dental } \\
\hline Dentista & 1,00 & 0,26 \\
\hline Familiares & $1,25(0,84-1,85)$ & \\
\hline \multicolumn{3}{|l|}{ Escovações diárias } \\
\hline 1 ou 2 & 1,00 & 0,42 \\
\hline 3 & $0,80(0,50-1,28)$ & \\
\hline 4 ou mais & $0,71(0,43-1,19)$ & \\
\hline \multicolumn{3}{|c|}{ Escovação na faculdade ou trabalho } \\
\hline Sim & 1,00 & 0,50 \\
\hline Às vezes & $0,98(0,65-1,44)$ & \\
\hline Não & $1,21(0,84-1,75)$ & \\
\hline \multicolumn{3}{|l|}{ Uso do fio dental } \\
\hline Sim & 1,00 & 0,21 \\
\hline Às vezes e/ou não & $1,42(1,05-1,92)$ & \\
\hline \multicolumn{3}{|c|}{ Flúor além da pasta dental } \\
\hline Sim & 1,00 & 0,54 \\
\hline Não & $1,12(0,78-1,58)$ & \\
\hline
\end{tabular}

A Odds ratio.

B Intervalo de confiança de $95 \%$

* Estatisticamente significativo. 
regularidade o dentista do que aqueles livres de cárie. Os que visitavam regularmente o dentista apresentaram risco 55\% maior de ter cárie ou restaurações do que os demais ( $O R=1,55$; IC 95\% 1,07-2,24).

Entre os casos (livres de cáries), o número dos que compravam a própria escova dental foi significativamente menor do que entre os controles ( $p=0,02$ ). Não comprar a própria escova dental esteve associado, também paradoxalmente, com menor risco de desenvolver cáries (OR =0,52; IC 95\% 0,30-0,90).

\section{Análise multivariada}

Com o objetivo de controlar potenciais fatores de confusão e investigar possíveis interações, realizou-se análise multivariada por regressão logística condicional (Breslow \& Day, 1980; Tabachinick \& Fidell, 1989; Victora et al., 1994). A inclusão das variáveis no modelo foi feita de acordo com a hierarquia do modelo teórico conceitual (Figura 1), tendo-se mantido, no modelo, as variáveis que alcançaram valor de $p$ menor ou igual a 0,20 . Os resultados concernentes a fatores socioeconômicos, familiares, comportamentais e dietéticos mostraram que apenas duas destas variáveis - "familiar livre de cáries" (primeiro nível de causalidade) e "consumo freqüente de açúcares" (terceiro nível) estiveram associadas ao desfecho (Petry et al., 1996). Para o presente estudo, estas duas variáveis foram mantidas no modelo em seu nível correspondente.

Inicialmente foram colocadas, no modelo de análise, as variáveis relacionadas à participação do profissional juntamente com a variável "familiar livre de cáries", tendo permanecido na equação apenas as consultas regulares ao dentista com odds ratio de 1,67 (IC 95\% 1,07$2,60)$. As demais variáveis relativas ao papel do dentista, conhecimento e atitudes pessoais não foram significativas. A variável "compra a própria escova", significativamente associada ao desfecho na análise inicial, perdeu a significância após o ajuste.

Mantidas apenas as variáveis significativas do bloco anterior, foram acrescentadas as do nível proximal ao modelo de análise, as quais são relacionadas aos cuidados com a higiene oral e ao uso de fluoretos, assim como à variável "consumo freqüente de açúcares". Neste bloco, a única variável significativamente associada ( $p=0,045$ ) com o desfecho foi o não uso de fio dental com oddsratio de 0,73 (IC 95\% 0,53- 0,99).

A Tabela 3 mostra os dados brutos e ajustados das duas variáveis significativamente relacionadas ao desfecho.

\section{Discussão}

Os estudos de casos e controles apresentam a vantagem de permitir o estudo de eventos raros, como a ausência total de história de cáries em adultos. Ao contrário dos estudos tradicionais de casos e controles, os casos - na presente investigação - foram pessoas sem qualquer comprometimento, ao passo que os controles foram pessoas com atividade cariosa.

Os resultados descritos acima evidenciam a possibilidade de um viés que é conhecido em Epidemiologia como "causalidade reversa" (Victora, 1992). O presente estudo revelou que as pessoas com história de cárie visitam o dentista com regularidade, em proporção bastante maior do que os livres de cárie (Tabela 1). Na análise por regressão logística condicional,

Tabela 3

Dados brutos e ajustados das variáveis significativamente associadas com o desfecho.

\begin{tabular}{|c|c|c|c|}
\hline Variável & $\begin{array}{l}\text { OR brutoA } \\
\text { (IC } 95 \%)^{B}\end{array}$ & $\begin{array}{l}\text { OR ajustadoC } \\
\text { (IC 95\%) }\end{array}$ & $\begin{array}{l}p \text { valor } \\
\text { (ajustado) }\end{array}$ \\
\hline \multicolumn{4}{|c|}{ Visitas regulares ao dentista } \\
\hline Sim & $0,64(0,44-0,97)$ & $0,59(0,38-0,93)$ & \\
\hline Não & 1,00 & 1,00 & 0,02 \\
\hline \multicolumn{4}{|l|}{ Uso do fio dental } \\
\hline $\operatorname{Sim}$ & 1,00 & 1,00 & 0,045 \\
\hline Às vezes e/ou não & $1,42(1,05-1,92)$ & $1,36(1,01-1,20)$ & \\
\hline
\end{tabular}

A Odds ratio (bruto).

B Intervalo de confiança de $95 \%$.

c O dds ratio (ajustado). 
constatou-se que a visita regular ao dentista torna maior a chance de cáries ou restaurações em cerca de $67 \%$. O mesmo foi observado quanto ao uso de fio dental, que igual mente aumentou esta chance. Conforme a hipótese de causalidade reversa, o fato de possuir cáries teria levado os indivíduos a procurar o dentista e a utilizar fio dental com maior freqüência, seguindo as recomendações profissionais.

Paralelamente, não deve ser descartada a hipótese de que recebam mais tratamentos restauradores - em especial, por questões relacionadas a critérios de diagnóstico - aqueles que consultam com maior freqüência. Estes achados estão de acordo com estudos que demonstram ser baixa a concordância entre profissionais quanto à detecção de lesões cariosas e ao plano de tratamento, bem como mostram relação positiva entre a troca de dentista e o número de restaurações recebidas (Elderton \& Nuttall, 1983; Davies, 1984).

Outro fator metodológico a ser tomado em conta é a relativa homogeneidade da amostra, constituída por estudantes universitários de baixa idade, que freqüentam faculdades particulares. Embora fossem observadas variações entre as práticas preventivas, não houve pessoa na amostra que, por exemplo, não possuísse escova dental. Se a atual pesquisa tivesse sido ampliada para todos os estratos sociais, talvez determinados resultados fossem diferentes.
Apesar dos achados paradoxais, chama a atenção o fato de que al gumas pessoas conseguem manter-se livres de cáries mesmo com hábitos preventivos inadequados. Note-se que $35 \%$ dos casos não visitam com regularidade o dentista, e, dentre os que o fazem, 33\% têm apenas uma consulta por ano. Além disso, cerca de $74 \%$ dos casos afirmaram não usar qualquer outro método de aplicação de flúor, a não ser o que está contido na pasta dental, e 18,5\% deles escovam os dentes apenas uma ou duas vezes por dia. Um número expressivo de casos $(56,3 \%)$ informou não usar fio dental ou fazê-lo eventualmente (Tabela 1). Mesmo sabendo-se ser a cárie uma doença multifatorial (Corbett \& Moore, 1976; Nikiforuk, 1985), é interessante ressaltar que fatores de proteção reconhecidamente eficazes - como hábitos de higiene oral (Wendt et al., 1994; Galan et al., 1995), e uso de fluoretos (Murray et al., 1991; Chadwick, 1994) - não estiveram associados à completa ausência de cáries.

Qual a possibilidade de que variáveis que reconhecidamente afetam o desenvolvimento de cáries não interfiram com sua total ausência? Existirão outros determinantes, talvez a nível genético ou dietético (Petry et al., 1996)?

Espera-se que tais indagações possam sugerir novas pesquisas com o propósito de investigar este grupo de afortunados que, apesar de cuidados precários, nunca desenvolveram cáries.
Referências

BRESLOW, N. E. \& DAY, N. E., 1980. Statistical Methods in Cancer Research. Vol. 1. The Analysis of Case Control Studies. Lyon: IARC.

CHADWICK, B. L., 1994. Products for prevention during orthodontics. British Journal of Orthodontics, 21:395-398

CORBETT, E. M. \& MOORE, W. J., 1976. Distribution of dental caries in ancient British populations. IV - The 19th century. Caries Research, 10:401414.

CORDON, J. A. \& GARRAFA, V., 1991. Prevenção versus preventivismo. Divulgação em Saúde para Debate, 6:10-16.

DAVIES, J. A., 1984. The relationship between change of dentist and treatment received in the General Dental Service. British Dentistry Journal, 157:322324. 
DOWNER, M. C., 1991. The improving dental health of United Kingdon adults and prospects for the future. British Dentistry Journal, 170:154-158.

ELDERTON, R. J. \& NUTTALL, N. M., 1983. Variation among dentists in planning treatment. British Dentistry Journal, 154:201-206.

EPI INFO, 1994. Epi Info Version 6.02. A Word Processing, Database, and Statistics System for Epidemiol ogy on Microcomputers. Geneva: World Health Organization.

FACCHINI, L. A., 1995. Trabalho Materno e Ganho de Peso Infantil. Pelotas: Editora Universitária.

FEHR, F. R. v.d., 1990. Caries status in the Nordic Countries and predictions of future trends. In: Caries Status in Europe and predictions of future trends (M. T. Marthaler, ed.). Caries Research, 24:381-396.

FEJERSKOV, O.; ANTOFT, P. \& GADEGAARD, E., 1982. Decrease in caries experience in Danish children and young adults in the 1970s. Journal of Dentistry Research, 61:1305-1310.

GALAN, D.; BRECX, M. \& HEATH, M. R., 1995. The oral health status of a population of communitydwelling older Canadians. Gerodontology, 12:4148.

ISTOÉ/ SENHOR, 1988. Dentes afiados. Istoé/Senhor, São Paulo, 993:95.

MALDONADO, G. \& GREENLAND, S., 1993. Simulation study of confounder-selection strategies. American Journal of Epidemiology, 138:923-936.

MURRAY, J. J.; RUGG-GUNN, A. J. \& JENKINS, G. N., 1991. Fluorides in Caries Prevention. Oxford: Butter-Worth-Heinemann Ltda.

NIKIFORUK, G., 1985. Understanding Dental Caries. I. Etiology and Mechanisms. Basic and Clinical Aspects. Basel: Karger.

ODONTONOTÍCIAS, 1988. Fio dental. Odontonotícias, São Paulo, 44:4.

ODONTONOTÍCIAS, 1992. O fenômeno das pastas no Brasil. Odontonotícias, São Paulo, 26:52.
PETRY, P. C.; VICTORA, C. G. \& SANTOS, I. S., 1996. Adul tos Livres de Cárie: Estudo de Casos e Controles sobre Fatores Sociais, Genéticos, Familiares e Dietéticos. Pelotas: Faculdade de Medicina, Universidade Federal de Pelotas.

SCHLESSELM AN, J. . ., 1982. Case-Control Studies Design, Conduct, Analysis. New York: Oxford University Press.

SPSS FOR WINDOWS, 1994. Statistical Packagefor the Social Sciences. Release 6.1 Chicago: SPSS Inc.

STAM M, J. W., 1991. The epidemiology of permanent tooth caries in the Americas. In: Risk Markers for Oral Diseases (N. W. Johnson, ed.), pp. 73-77, Cambridge: Cambridge University Press.

TABACHNICK, B. G. \& FIDELL, L. S., 1989. Using MultivariateStatistics. New York: Harper Collins.

THYLSTRUP, A. \& BRUUN, C., 1992. The use of dentifrices in the treatment of dental caries. In: Clinical and Biological Aspects of Dentifrices (G. Embery \& G. Rolla, eds.), pp. 131-143, Oxford: Oxford University Press.

VICTORA, C. G., 1992. Case-control studies in maternal and child health. In: Measurement of Maternal and Infant Mortality, Morbidity and Health Care. Interdisciplinary Approaches (J. T. Boerma, ed.), pp. 85-108, Liège: Deaouaux-Ordina.

VICTORA, C. G.; FUCHS, S. C.; FLORES, J. A. C.; FONSECA, W. \& KIRKWOOD, B. R., 1994. Risk factors for pneumonia among children in a Brazilian metropolitan area. Pediatrics, 93:977-985.

WENDT, L. K.; HALLONSTEN, A. L.; KOCH, G. \& BIRKHED, D., 1994. Oral hygiene in relation to caries development and immigrant status in infants and toddlers. Scandinavian Journal of Dentistry Research, 102:269-273.

WHO (World Health Organization), 1987. Oral Health Surveys - Basic Methods. Geneva: WHO. 